\title{
Lattice simulations of Born-Infeld non-linear QED
}

\section{K. Sinclair ${ }^{* \dagger}$}

HEP Division, Argonne National Laboratory, 9700 South Cass Avenue, Argonne, IL 60439, USA

E-mail: dks@hep.anl.gov

\section{J. B. Kogut}

Department of Energy, Division of High Energy Physics, Washington, DC 20585, USA

and

Dept. of Physics - TQHN, Univ. of Maryland, 82 Regents Dr., College Park, MD 20742, USA

E-mail: jbkogut @umd.edu

Born-Infeld non-linear electrodynamics was introduced to render the self energy of a point particle finite. It has recently been revived as a field theory for branes and strings. We quantize this theory on a Euclidean space-time lattice, using Metropolis Monte-Carlo simulations to measure the properties of the quantum field theory. Lüscher-Weisz methods are used to measure the electromagnetic fields from a static point charge. The $\mathbf{D}$ field from a point charge appears to be identical to that for the normal Maxwell Lagrangian. The $\mathbf{E}$ field is enhanced by quantum fluctuations, and shows short distance screening as it does in the classical theory.

XXIIIrd International Symposium on Lattice Field Theory

25-30 July 2005

Trinity College, Dublin, Ireland

\footnotetext{
*Speaker.

${ }^{\dagger}$ This work was supported by the U.S. Department of Energy, Division of High Energy Physics, Contract W-31-109-ENG-38.

¥ Supported in part by NSF grant NSF PHY03-04252.
} 


\section{Introduction}

The $n+1$ dimensional Born-Infeld (non-linear electrodynamics) action [1] 2] is:

$$
S=b^{2} \int d^{n+1} x\left[1-\sqrt{-\operatorname{det}\left(g_{\mu \nu}+\frac{1}{b} F_{\mu \nu}\right)}\right] .
$$

This has seen a revival as a theory of strings and branes [3, 4, , , 6, 7, 7. Choosing $n=9$, and dimensionally reducing this action from $9+1$ to $p+1$ dimensions describes a $p$-brane. The $9-p$ additional components of $A_{\mu}$ are identified with the transverse components of the string/brane.

Most of the serious work on these theories has dealt with their classical behaviour [8]. We are simulating these quantum theories on the lattice. We are starting with the simplest case where $n=p=3$, the original Born-Infeld modification of electrodynamics, designed to make the self energy of a point charge finite.

Section 2 reviews the classical Born-Infeld theory. In section 3 we indicate how this is ported to the lattice allowing Monte-Carlo simulations of the quantum theory. Section 4 details our simulations and preliminary results. A discussion of our results and conclusions are given in section 5 .

\section{Classical Born-Infeld electrodynamics in Minkowski space-time}

This section summarises those results in references [9, 10, 11, 12] which are relevant for our investigations.

Evaluating the determinant in equation 1.1 the Lagrangian in $3+1$ dimensions is

$$
\mathscr{L}=b^{2}\left[1-\sqrt{1-b^{-2}\left(\boldsymbol{E}^{2}-\boldsymbol{B}^{2}\right)-b^{-4}(\boldsymbol{E} \cdot \boldsymbol{B})^{2}}\right] .
$$

One can now define $\boldsymbol{D}$ and $\boldsymbol{H}$ by

$$
\begin{aligned}
& \boldsymbol{D}=\frac{\partial \mathscr{L}}{\partial \boldsymbol{E}}=\frac{\boldsymbol{E}+b^{-2}(\boldsymbol{E} \cdot \boldsymbol{B}) \boldsymbol{B}}{\sqrt{1-b^{-2}\left(\boldsymbol{E}^{2}-\boldsymbol{B}^{2}\right)-b^{-4}(\boldsymbol{E} \cdot \boldsymbol{B})^{2}}} \\
& \boldsymbol{H}=\frac{\partial \mathscr{L}}{\partial \boldsymbol{B}}=\frac{\boldsymbol{B}-b^{-2}(\boldsymbol{E} \cdot \boldsymbol{B}) \boldsymbol{E}}{\sqrt{1-b^{-2}\left(\boldsymbol{E}^{2}-\boldsymbol{B}^{2}\right)-b^{-4}(\boldsymbol{E} \cdot \boldsymbol{B})^{2}}} .
\end{aligned}
$$

Interaction with charged particles is implemented, as usual, by adding a term $j_{\mu} A^{\mu}$ to the Lagrangian. In terms of $\boldsymbol{E}, \boldsymbol{B}, \boldsymbol{D}$ and $\boldsymbol{H}$, the equations of motion are the standard Maxwell equations. The non-linearity is hidden in equations 2.2 .

For a static point charge $\rho=e \delta^{3}(\boldsymbol{r})$ the electric fields are

$$
\begin{aligned}
& \boldsymbol{D}=\frac{e}{4 \pi r^{2}} \hat{\boldsymbol{r}} \\
& \boldsymbol{E}=\frac{e}{4 \pi} \frac{\hat{\boldsymbol{r}}}{\sqrt{r^{4}+r_{0}^{4}}}
\end{aligned}
$$

where $\hat{\boldsymbol{r}}=\frac{\boldsymbol{r}}{r}$ and $r_{0}=\sqrt{\frac{|e|}{4 \pi b}}$. Hence the $\boldsymbol{D}$ field for a static point charge is identical to the Maxwell solution, while the $\boldsymbol{E}$ field is screened at short distances. 


\section{Lattice Born-Infeld quantum-electrodynamics}

The Euclidean space action for Born-Infeld QED

$$
S=b^{2} \int d^{4} x\left[\sqrt{1+b^{-2}\left(\boldsymbol{E}^{2}+\boldsymbol{B}^{2}\right)+b^{-4}(\boldsymbol{E} \cdot \boldsymbol{B})^{2}}-1\right]
$$

is positive. Hence it can be simulated using Monte-Carlo methods.

On the lattice we use the non-compact formulation:

$$
F_{\mu v}\left(x+\frac{1}{2} \hat{\mu}+\frac{1}{2} \hat{v}\right)=A_{v}(x+\hat{\mu})-A_{v}(x)-A_{\mu}(x+\hat{v})+A_{\mu}(x)
$$

and average over the 16 choices of 6 plaquettes associated with each lattice site. We also define $\beta=b^{2} a^{4}$ where $a$ is the lattice spacing. Simulations are performed using the Metropolis MonteCarlo method [13].

We measure the $\boldsymbol{E}$ and $\boldsymbol{D}$ fields due to a static point charge. This point charge $e$ is introduced by including a Wilson Line (Polyakov Loop) $W(\boldsymbol{x})$.

$$
W(\boldsymbol{x})=\exp \left\{i e \sum_{t}\left[A_{4}(\boldsymbol{x}, t)-\frac{1}{N_{x} N_{y} N_{z}} \sum_{\boldsymbol{y}} A_{4}(\boldsymbol{y}, t)\right]\right\}
$$

The second ('Jellium') term is needed, since a net charge would be inconsistent with periodic boundary conditions on $A_{\mu}$. $\langle\boldsymbol{E}\rangle$ and $\langle\boldsymbol{D}\rangle$ in the presence of this charge are given by

$$
\begin{aligned}
i\langle\boldsymbol{E}\rangle_{\rho}(\boldsymbol{y}-\boldsymbol{x}) & =\frac{\langle\boldsymbol{E}(\boldsymbol{y}, t) W(\boldsymbol{x})\rangle}{\langle W(\boldsymbol{x})\rangle} \\
i\langle\boldsymbol{D}\rangle_{\rho}(\boldsymbol{y}-\boldsymbol{x}) & =\frac{\langle\boldsymbol{D}(\boldsymbol{y}, t) W(\boldsymbol{x})\rangle}{\langle W(\boldsymbol{x})\rangle} .
\end{aligned}
$$

Since $W$ is complex, there is a sign problem, which causes $\langle W(\boldsymbol{x})\rangle$ to fall exponentially with $N_{t}$. We use the method of Lüscher and Weisz [14] (Parisi, Petronzio and Rapuano [15]) with thickness 1 and 2 timeslices to overcome this exponential factor.

\section{Simulations and Results}

We have performed preliminary simulations of 500,000 10-hit Metropolis sweeps of the lattice at $\beta=100,1.0,0.01,0.0001$ and 100,000 sweeps at $\beta=5,2,0.5,0.2,0.1$, making measurements every 100 sweeps. We measured the $\boldsymbol{E}$ and $\boldsymbol{D}$ fields for on axis separations from the point charge. Figure 1 shows the expectation value of the Wilson lines (Polyakov loops) obtained from these simulations. Note that the value falls rapidly with increasing $e$. The rate of falloff also increases with increasing non-linearity (decreasing $\beta$ ). Note also the small relative errors, even when the magnitude has fallen 8 orders of magnitude, which shows the effectiveness of the Lüscher-Weisz method.

Figure 2 shows the ratio of the $\boldsymbol{E}$ field in the direction of the separation from the charge to the $\boldsymbol{D}=\boldsymbol{E}$ field for the free field (Maxwell) theory, at the minimum separation $(Z=0.5)$, in the limit of zero charge. At large $\beta$, where the Born-Infeld theory asymptotes to the Maxwell theory, this ratio 


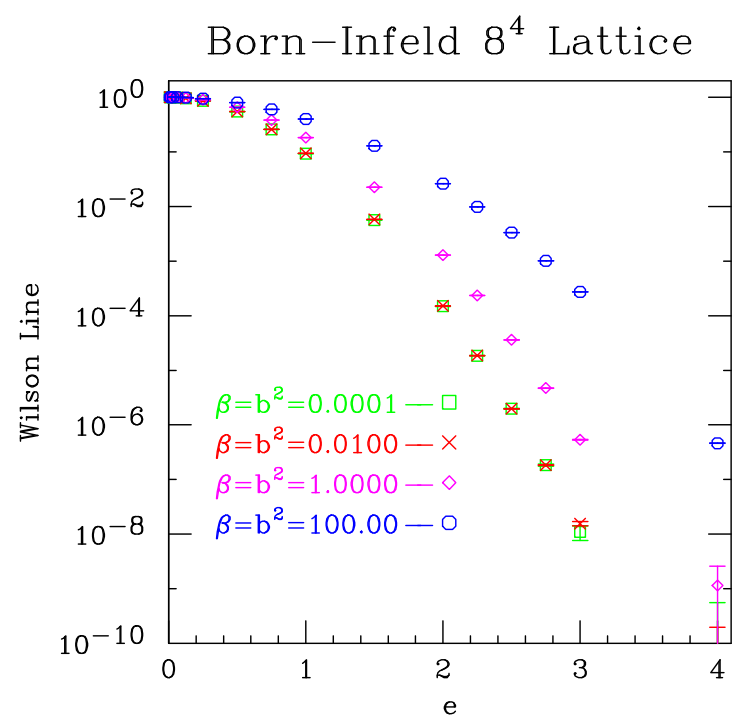

Figure 1: Wilson Lines as functions of charge $e$, for a range of $\beta=a^{2} b^{2}$.

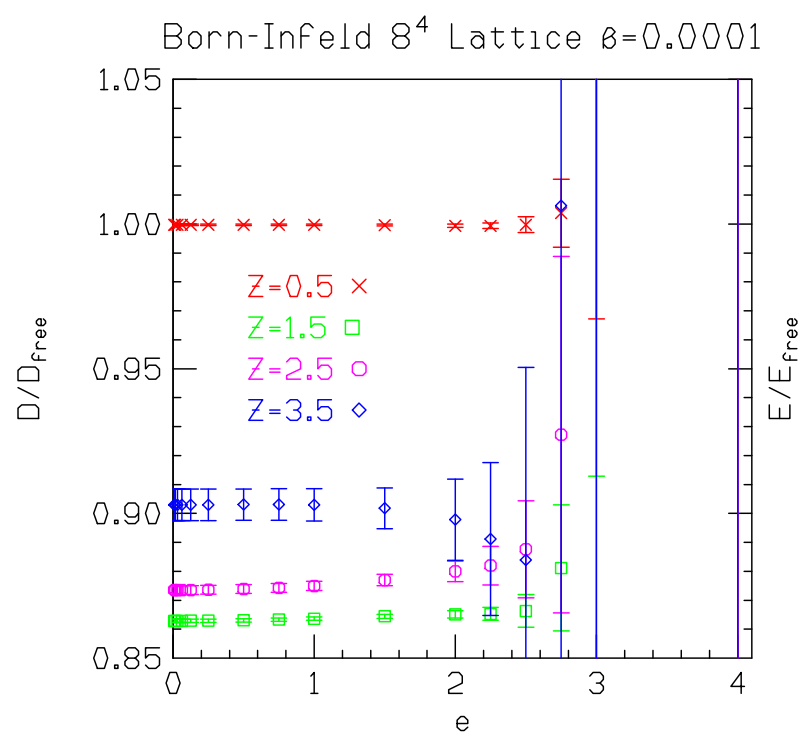

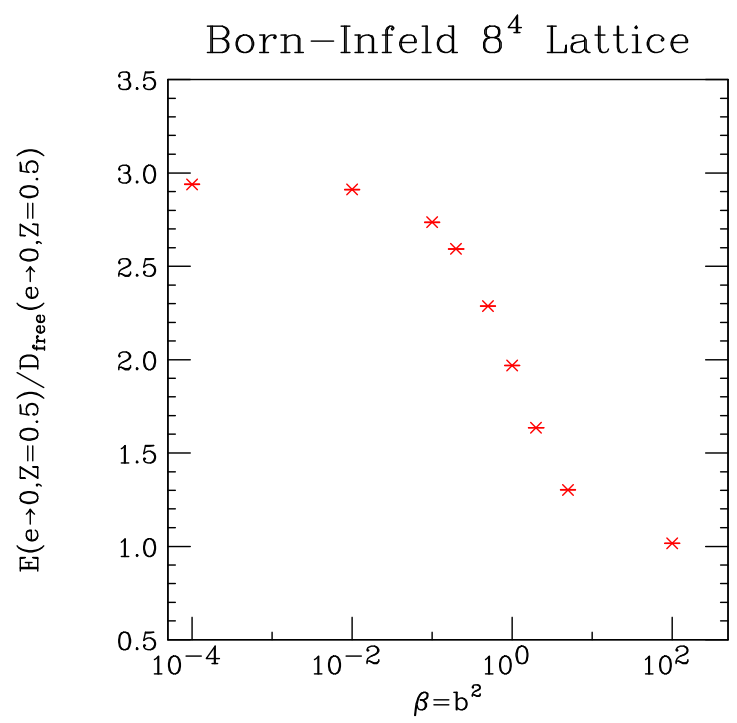

Figure 2: $E / D$ ratio at minimum separation for $e \rightarrow$ 0 as a function of $\beta$.

$$
\text { Born-Infeld } 8^{4} \text { Lattice } B=0.0001
$$

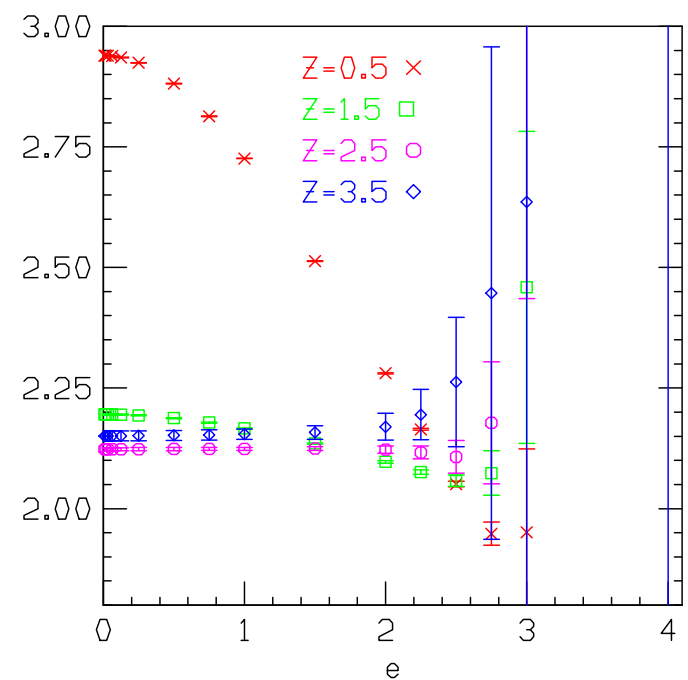

Figure 3: a) $\boldsymbol{D}$ fields at distance $Z$ from the point charge $e$, scaled by the free field (Maxwell) values, for $\beta=0.0001$. b) $\boldsymbol{E}$ fields at distance $Z$ from the point charge $e$, scaled by the free field (Maxwell) values, for $\beta=0.0001$.

approaches 1. Classically, this ratio is 1 for all values of $b$. Quantum fluctuations cause this ratio to increase with increasing non-linearity (decreasing $\beta$ ), approaching a value close to 3 for small $\beta$.

In figure $3 \mathrm{a}$, we plot the $\boldsymbol{D}$ fields scaled by their free field values at each separation, as a function of charge, for $\beta=0.0001$, where the non-linearity is large, and the $\boldsymbol{D}$ field comes almost entirely from the $(\boldsymbol{E} \cdot \boldsymbol{B}) \boldsymbol{B}$ term in its definition. The fact that this ratio is still 1 for all $e$ at minimal separation is because $\boldsymbol{D}$ still obeys $\boldsymbol{\nabla} \cdot \boldsymbol{D}=\rho$, combined with cubic symmetry. Because we do not have full rotational symmetry on the lattice $\boldsymbol{\nabla} \cdot \boldsymbol{D}=\rho$ is insufficient to make this ratio 1 at other 
separations. The fact that this ratio is never more than $15 \%$ from 1 suggests that it would be 1 if we had rotational symmetry. However, we would expect rotational symmetry to be restored at large distances, which is why the ratio is closer to 1 for larger separations. Figure $3 \mathrm{~b}$ is a similar graph for the $\boldsymbol{E}$ field. As well as showing the effects of quantum fluctuations as in figure 2 , the $\boldsymbol{E}$ field is clearly screened at short distances as $e$ increases, similar to what is seen in the classical theory.

What is different from the classical theory is that classically the screening length continues to increase with decreasing $b$. The quantum theory approaches a limit as $\beta \rightarrow 0$.

\section{Discussion and conclusions}

We have succeeded in using lattice Monte-Carlo methods to extract non-perturbative physics from Born-Infeld electrodynamics, quantized using the Euclidean-time functional integral approach. The on-axis (quantum) electrostatic fields of a point charge are measured as functions of the charge $e$ introduced as a Wilson Line. The approach of Lüscher and Weisz, which reduces these measurements from an exponential- to a polynomial-time problem, was essential for extracting these quantities.

In the classical field-theory $E / D \rightarrow 1$ as $e \rightarrow 0$. For the quantum theory $E / D$ increases from 1 as the nonlinearity is increased indicating that the dielectric constant $\varepsilon<1$.

As $|e|$ is increased, the $\boldsymbol{E}$ field is screened at short distances. Screening increases with $|e|$ and with increasing nonlinearity $\left(\beta\right.$ or $b$ ). The screening length $r_{0}$ appears to increase as $\sqrt{|e|}$ as for the classical theory. $\boldsymbol{D}$ shows no such screening and appears to independent of the non-linearity, while $\boldsymbol{D} / e$ appears independent of $e$.

Unlike the classical theory, where the screening length diverges as $b \rightarrow 0$, the quantum theory approaches a fixed-point field theory as $\beta=b^{2} a^{4} \rightarrow 0$. This conformal field theory has Euclidean Lagrangian $\mathscr{L}_{E}=\frac{1}{4}|\boldsymbol{E} . \boldsymbol{B}|$ and Hamiltonian $\mathscr{H}=|\boldsymbol{D} \times \boldsymbol{B}|$ [9, 16].

Normally, Born-Infeld QED is considered as an effective field theory with a momentum cutoff. However, as this cutoff $\rightarrow \infty$ it approaches the above fixed-point theory. If this fixed-point field theory is non-trivial, it would serve to define Born-Infeld QED without a cutoff.

These first simulations were performed on $8^{4}$ lattices. We are extending these simulations to larger lattices. We then plan to study those $p$-brane theories obtained by dimensional reduction of $n+1$ dimensional Born-Infeld theories to determine if the quantized theories continue to show string/brane dynamics.

\section{Acknowledgements}

Our simulations are currently running on the Rachael supercomputer at the Pittsburgh Supercomputer Center.

\section{References}

[1] M. Born, On the Quantum Theory of Electromagnetic Field Proc. Roy. Soc. Lond. A 143 (1934) 410.

[2] M. Born and L. Infeld, Foundations Of The New Field Theory, Proc. Roy. Soc. Lond. A 144 (1934) 425. 
[3] E. S. Fradkin and A. A. Tseytlin, Nonlinear Electrodynamics From Quantized Strings, Phys. Lett. B 163 (1985) 123.

[4] R. G. Leigh, Dirac-Born-Infeld Action From Dirichlet Sigma Model, Mod. Phys. Lett. A 4 (1989) 2767.

[5] M. Aganagic, C. Popescu and J. H. Schwarz, Gauge-invariant and gauge-fixed D-brane actions, Nucl. Phys. B 495 (1997) 99 [arXiv:hep-th/9612080].

[6] G. W. Gibbons, Born-Infeld particles and Dirichlet p-branes, Nucl. Phys. B 514 (1998) 603 [arXiv:hep-th/9709027].

[7] A. A. Tseytlin, Born-Infeld action, supersymmetry and string theory, [arXiv:hep-th/9908105].

[8] C. G. Callan and J. M. Maldacena, Brane dynamics from the Born-Infeld action, Nucl. Phys. B 513 (1998) 198 [arXiv:hep-th/9708147].

[9] I. Bialynicki-Birula, Nonlinear Electrodynamics: Variations On A Theme By Born And Infeld, In *Jancewicz, B. ( Ed.), Lukierski, J. ( Ed.): Quantum Theory Of Particles and Fields*, World Scientific(1983) 31-48.

[10] D. Chruscinski and J. Kijowski, Generation of a dipole moment by external field in Born-Infeld non-linear electrodynamics, Compt. Rend. Acad. Sci. (Ser. II) 324 (1997) 435 [arXiv:hep-th/9712101].

[11] D. Chruscinski, Point charge in the Born-Infeld electrodynamics, Phys. Lett. A 240 (1998) 8 [arXiv:hep-th/9712161].

[12] D. Chruscinski, Canonical formalism for the Born-Infeld particle, J. Phys. A 31 (1998) 5775 [arXiv:hep-th/9803100].

[13] N. Metropolis, A. W. Rosenbluth, M. N. Rosenbluth, A. H. Teller and E. Teller, Equation Of State Calculations By Fast Computing Machines, J. Chem. Phys. 21 (1953) 1087.

[14] M. Luscher and P. Weisz, Locality and exponential error reduction in numerical lattice gauge theory, JHEP 0109 (2001) 010 [arXiv:hep-lat/0108014].

[15] G. Parisi, R. Petronzio and F. Rapuano, A Measurement Of The String Tension Near The Continuum Limit, Phys. Lett. B 128 (1983) 418.

[16] D. Chruscinski, Strong field limit of the Born-Infeld p-form electrodynamics, Phys. Rev. D 62 (2000) 105007 [arXiv:hep-th/0005215]. 